\title{
Protein-Polymer Supramolecular Assemblies: A Key Combination for Multifunctionality
}

\author{
Cornelia G. Palivan*, Xiaoyan Zhang, and Wolfgang Meier*
}

\begin{abstract}
D/3D structures resulting from self-assembly of amphiphilic block copolymers can be combined with bioactive compounds, such as proteins and enzymes, to create supramolecular assemblies with specific desired properties and functionality. Chemical tuning of the architecture and properties of supramolecular assemblies to accommodate sensitive biomolecules allows the development of new soft hybrid materials that benefit from the robustness of polymers and from the functionality of biomolecules. The encapsulation/insertion of biomolecules (enzymes, mimics, proteins) in self-assembling block copolymer vesicles enables design of 'nanoreactors' both in solutions and at surfaces for highly diverse applications, ranging from production of antibiotics to creation of artificial organelles. When membrane proteins are inserted into polymer membranes, it is possible to generate functional membranes or active surfaces with a rapid and specific response. In addition, the selective binding of ligand-terminated polymers holds potential for targeted delivery of drugs, or for immobilization on solid support, to provide functional 3D assemblies on an extended surface.
\end{abstract}

Keywords: Amphiphilic copolymers · Enzymes · Nanoreactors · Polymer membranes · Proteins

\section{Introduction}

In various domains, including catalysis, medicine, environmental science, and electronics, new systems are actively being sought to address the need for stable and multifunctional membranes for nanometer-scale molecular devices, and for rapid and sensitive identification of specific molecules. A smart strategy for developing such systems consists of combining synthetic membranes able to mimic biological membranes with specific biological molecules, such as peptides, enzymes and proteins. ${ }^{11]}$ Such polymer membranes are generated either as boundaries of 3D assemblies (capsules, vesicles) or as planar structures formed on solid supports via polymerization, grafting or spreading. ${ }^{[2]}$

Amphiphilic polymers are generally regarded as higher molar mass homologues of lipids. If these polymers contain the appropriate hydrophobic-hydrophilic domains, they will self-assemble in aqueous solution to form a variety of supramolecular assemblies (micelles, vesicles, tubes, and combinations thereof). ${ }^{[3]}$ Amphiphilic

\footnotetext{
${ }^{*}$ Correspondence: Prof. Dr. W. Meier; Prof. Dr. C. G. Palivan University of Basel

Department of Chemistry

Klingelbergstrasse 80

$\mathrm{CH}-4056$ Basel

Tel.: +4161267 3802; +41612673839

E-mail: wolfgang.meier@unibas.ch;

cornelia.palivan@unibas.ch
}

block copolymers frequently form larger assemblies with considerably higher mechanical stability than conventional lipid bilayers. ${ }^{[4]}$ Block copolymers' properties, such as hydrophobic/hydrophilic ratio, molecular mass of each block, and phase behavior (e.g. fluid or glassy) control the mechanical stability and flexibility of the membrane. ${ }^{[2,5]}$

Recently the 'library' of polymers that generate membranes mimicking biomembranes was considerably extended with polysaccharides, ${ }^{[6]}$ peptides, ${ }^{[7]}$ zwitterionic $^{[8]}$ and stimuli-responsive polymers. ${ }^{[9]}$ Symmetric membranes based on amphiphilic $\mathrm{AB}$ and ABA block copolymers and asymmetric ones generated by $\mathrm{ABC}$-type triblock copolymers have been reported. ${ }^{[2]}$ While the synthesis

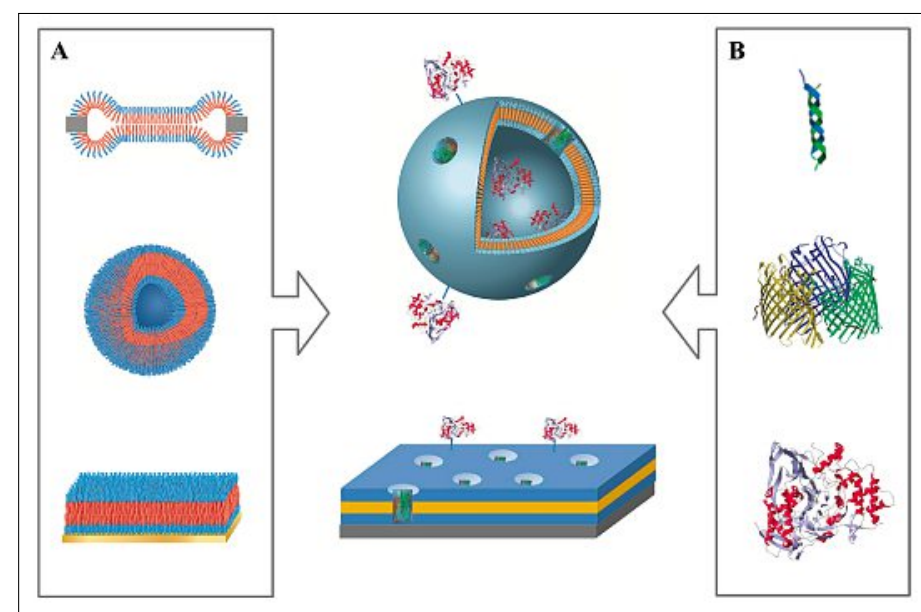

and self-assembly of $\mathrm{ABC}$ triblock copolymers such as poly(ethylene oxide)block-poly(dimethylsiloxane)-block-poly(2-methyloxazoline) (PEO- $b$-PDMS- $b$ PMOXA) have been reported, ${ }^{[10]}$ very few studies contain detailed characterisation of their morphology. ${ }^{[11]}$ Such asymmetric membranes are of particular interest due to their ability to undergo membrane inversion, which has significant potential for novel applications.[12]

Our group is interested in the development of new membrane structures, specifically spherical compartments and planar membranes that can accommodate biological molecules by immobilization at the surface, by insertion inside the membrane, and/or by encapsulation in the cavity of 3D compartments (Fig. 1). ${ }^{[2]}$

Fig. 1. A schematic image of the combination of polymer membranes $(A)$ with biomolecules (B). The polymer membrane can be a bi- or trilayer with hydrophilic (blue) and hydrophobic (red/ yellow) domains, respectively. The shape of such a membrane can either be 2D (planar) or 3D (boundary of vesicles). 
Here we will present hybrid systems based on combining bioactive molecules (enzymes, proteins, mimics) with polymer membranes that serve as stable boundaries for nanocompartments and as bilayers on solid supports. These hybrid systems have multifunctionality and responsiveness that help target their use for desired applications.

\section{Polymer Membranes of Amphiphilic Copolymers}

The versatility of polymer chemistry allows the tailoring of properties and potential functions of the resulting membranes in order to support specific applications, such as targeting approaches in therapeutics, immobilization on solid supports, or responsive behaviors. The chemical nature of the basic polymer building blocks has been exploited for coupling functional groups, such as peptides and proteins, ${ }^{[13]}$ ligands, ${ }^{[14]}$ aptamers and antibodies, ${ }^{[15]}$ which are suited for molecular recognition at the surface of vesicles. In recent years, we and others have successfully applied molecular recognition interactions for cell targeting and/or immobilisation of block copolymer vesicles onto surfaces. ${ }^{[15,16]}$ Vesicles of poly(dimethylsiloxane)block-poly(2-methyloxazoline) (PDMS$b$-PMOXA) diblock copolymers were conjugated with trastuzumab antibody for a fast and specific uptake into SKBR3 cells, ${ }^{[15]}$ whilst Ni-NTA groups exposed at the surface of poly(butadiene)-blockpoly(ethylene glycol) (PB- $b$-PEG) vesicles served for binding His-tag proteins in the micromolar range. ${ }^{[14]}$ Interestingly, the distribution of metal-NTA groups on the surface of vesicles allowed the formation of a homogeneous biomolecule layer upon binding His-tags or His-tag proteins, due to a molecular recognition interaction.

A biological molecule is combined with a polymer membrane by addition to the system before, during or after the membrane formation. ${ }^{[17]}$ Remarkably, synthetic block copolymer membranes allow a functional insertion of membrane proteins, despite being significantly thicker than lipid membranes. ${ }^{[18]}$ For example, transmembrane transport has been demonstrated by successful incorporation of pore proteins LamB, OmpF, maltoporin, and aquaporin into polymer membranes (Fig. 2A). ${ }^{[2,19]}$ Such systems offer new possibilities for bioconversion, filter devices and virus 'traps', as has been successfully proved by development of new highly selective water-permeable polymer membranes by inserting the water channel Aquaporin Z from E. Coli. [20]

It is interesting to note that after removal of the lipid component NADH-ubiquinone

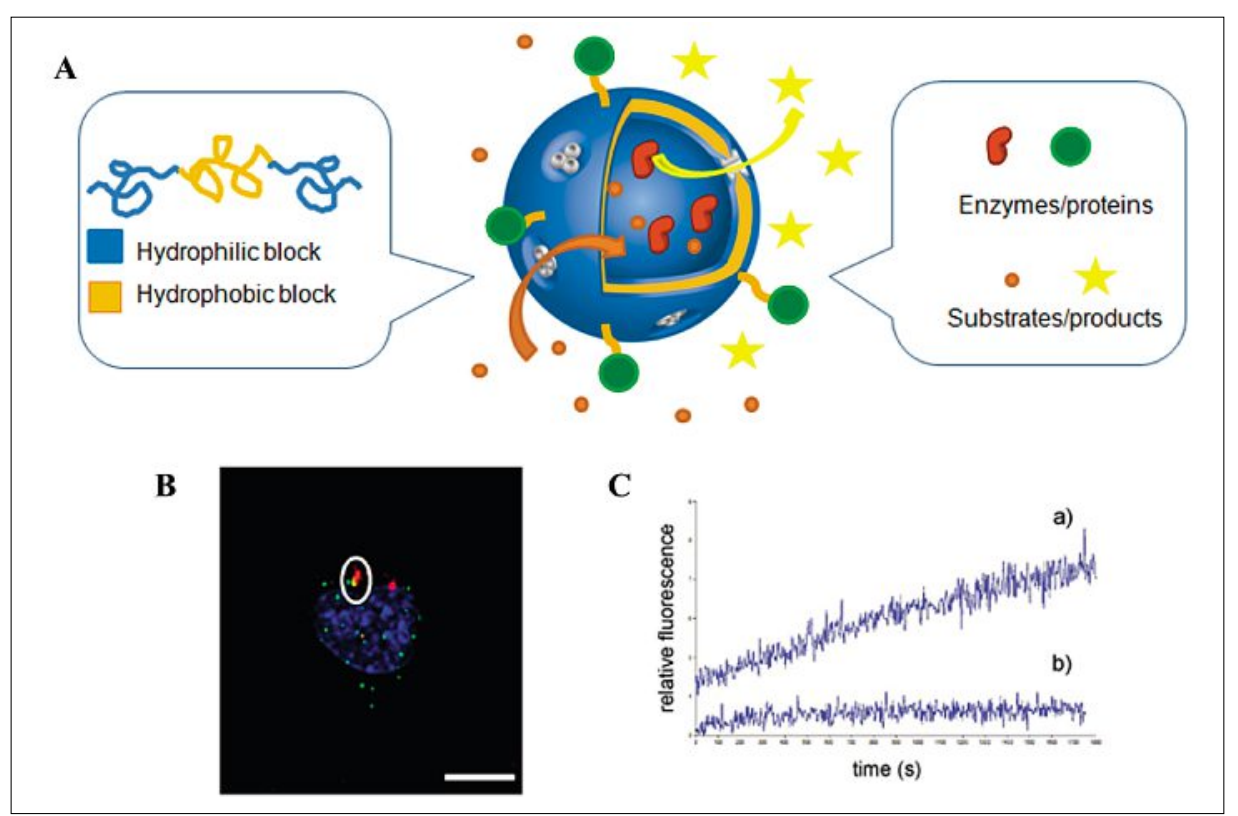

Fig. 2. (A) Schematic representation of a nanoreactor based on encapsulation of enzymes and insertion of channel proteins in their membrane, (B) CLSM image of HeLa cells incubated for $24 \mathrm{~h}$ with artificial peroxisomes (APs) (blue: nucleus stained with Hoechst, green: endosome/lysosome stained with $\mathrm{pH}$-rodo, red: artificial peroxisomes) (Scale bar $=5 \mu \mathrm{m})$, (C) Real time ROS detoxification kinetics of APs in: a) cells treated with pyocyanin, and b) cells pre-treated with APs ( $8 \mathrm{~h})$ followed by treatment with pyocyanin.

oxidoreductase recovered its activity in the presence of poly(2-methyloxazoline)block-poly(dimethylsiloxane)-blockpoly(2-methyloxazoline) (PMOXA- $b$ PDMS-b-PMOXA). ${ }^{[21]}$ Polymeric bilayers having specific molecular properties provided an environment that allowed membrane proteins to adopt an active conformation. NADH-ubiquinone oxidoreductase activity served to modulate electron transfer across the polymer membrane, and exhibited obvious dependence on the molecular composition of the block copolymer. This could potentially be exploited for the design of biomimetic batteries or fuel cells, or to perform specific chemical reactions inside or across polymer membranes.

\section{Nanoreactors}

Recently we introduced the concept of polymer nanoreactors that use polymer vesicles with encapsulated active compounds (enzymes, proteins, mimics) as nanometer-sized reaction compartments (Fig. 2). ${ }^{[22]}$ Polymer compartments play a dual role with active compounds: to shield them from the outside environment, for example, against proteolytic attack, and to allow them to act in situ. In addition, the compartments serve to co-encapsulate different active compounds, in order to support cascade reactions. To achieve persistent continuous reactions requires selective permeability of the nanoreactor membrane, in order to exchange substrates and products with the environment. Typically this requires a particular membrane permeability, which can be induced by using appropriately designed block copolymers ${ }^{[23]}$ that form nanoporous membranes, or by inserting channel proteins into the vesicle walls (Fig. 2A). ${ }^{[24]}$ A variety of nanoreactors have been reported as a result of changing the active compounds, and therefore the intended applications. ${ }^{[22-25]}$

For example, we introduced the concept of antioxidant nanoreactors to combat reactive oxygen species, such as superoxide radicals ${ }^{[23]}$ and peroxinitrites, ${ }^{[26]}$ which are well known to be involved in pathologies associated with oxidative stress. By encapsulating superoxide dismutase or its mimics inside the cavity of PMOXA- $b$-PDMS$b$-PMOXA vesicles, the active compounds detoxified superoxide radicals in situ. ${ }^{[23,27]}$ The in situ detoxification reaction was possible because of the intrinsic permeability of PMOXA- $b$-PDMS- $b$-PMOXA membrane to superoxide radicals.

By specific selection of hemoglobin, we successfully designed nanoreactors with dual functionality: transport of oxygen to serve as artificial blood, and simultaneous detoxification of peroxinitrites. ${ }^{[26]}$ Indeed, hemoglobin preserved its dual activity and provided multifunctionality to the nanoreactors, when encapsulated in polymer vesicles having a membrane rendered permeable by insertion of channel porins, OmpF. The insertion of channel proteins such as OmpF represents an elegant approach for the design of nanoreactors with membrane permeable to substrates/products, which allows in situ 
enzymatic reactions. Various membrane proteins such as LamB, FhuA, Tsx, and AqpZ have been successfully reconstituted in PMOXA- $b$-PDMS- $b$-PMOXA membranes, and thereby supported the movement of different molecules (water, NADH, nucleotides, DNA, enzyme substrates) through the polymeric membrane. In this respect the functional insertion of AqpZ in the polymer membrane led to the production of efficient and sustainable water treatment membranes with productivity at least one order of magnitude higher than that of the existing salt-rejecting polymer membranes. ${ }^{[20]}$

Such nanoreactors are robust, and efficient for the production of specific drugs in a desired location, for example in implants. By encapsulation of the enzyme penicillin acylase in PMOXA- $b$-PDMS- $b$-PMOXA amphiphilic triblock copolymer, the nanoreactors serve for local and controlled production of antibiotics.[28] The amount of antibiotic produced by the nanoreactors and then released was able to inhibit bacterial growth on agar plates. Because our system synthesizes an antibiotic on demand, with control of the amount as well as the timing of synthesis and release, it is expected to lead to increased therapeutic efficacy, lower toxic side effects, and reduced costs.

A step further in increasing the complexity for specific applications is to co-encapsulate active compounds inside nanoreactors, to support cascade reactions. ${ }^{[29]}$ Superoxide dismutase and lactoperoxidase were shown to participate in sequential reactions in situ in the nanovesicle cavity, transforming superoxide radicals to molecular oxygen and water. The diffusion of LPO substrate/products was facilitated by insertion of OmpF. ${ }^{29 a}$ ] This complex antioxidant nanoreactor was optimized by changing the enzymes to superoxide dismutase and catalase in tandem, increasing the encapsulation efficiency and modulation of membrane permeability. The optimized nanoreactor was active for more than 48 hours after up-take by various cell lines, thus mimicking peroxisomes, natural organelle known for their role in detoxification of reactive oxygen species (Fig. 2B and C). ${ }^{[30]}$ Such artificial organelles represent a new concept for cellular implants able to produce bioactive molecules at a subcellular level.

\section{Solid-supported Polymer Membranes}

Amphiphilic block copolymers are suitable for preparing surface-immobilized membranes, also known as solid-supported polymer membranes. As the membrane is attached to a solid surface, the resulting system has improved mechanical stability compared to isolated membranes. ${ }^{[31]}$ Solid-supported polymer membranes are similar to solid-supported lipid membranes in architecture and fluidity, but outperform them in thickness and stability. A variety of strategies are available for immobilizing polymer membranes on surfaces: synthetic methods (grafting-to, graftingfrom), fusion of vesicles on solid supports, and transfer of monolayers. They can be advantageously combined with membrane modifications or the insertion/attachment of active compounds to generate highly 'active surfaces'.[2] The most common architectures for solid-supported polymer membranes are: i) surface immobilized planar membranes (Fig. 3A), ${ }^{[32]}$ and ii) surface-tethered vesicles (Fig. 3B). [33]

The 'grafting-to' technique for preparation of polymer solid-supported membranes consists of anchoring previously synthesized polymers to a substrate via chemical or physical interactions.[34] The major drawback of the 'grafting-to' method is the lack of full surface coverage due to excluded volume effects of the polymer chains on the surface, and uncontrollable orientation of the chains during the anchoring procedure. Whilst experimentally simple, samples prepared using this approach are frequently not reproducible. ${ }^{[35]}$ To overcome this disadvantage, the 'grafting-from' method involves a covalent immobilization of small initiator molecules on the surface, followed by step-by-step polymerization to obtain a membrane with defined architecture. The first successful attempt using this approach was the covalent immobilization of the poly(2-hydroxyethyl methacrylate)-block-poly(n-butyl methacrylate)-block-poly(2-hydroxyethyl methacrylate) (PHEMA- $b$-PBMA- $b$ PHEMA) membrane on gold and silicon solid supports by using surface-initiated atom transfer radical polymerization (ATRP). ATRP allows a controlled step-

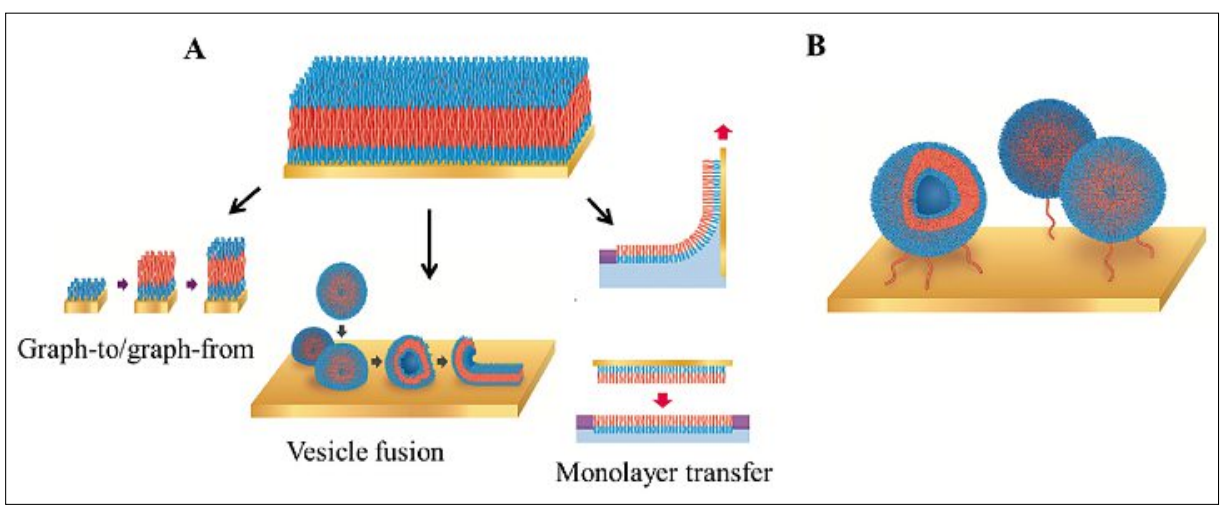

Fig. 3. Scheme for the two most common configurations for solid-supported polymer membranes, (A) surface immobilized planar membranes, which are able to be prepared by graph-to/ graph-from, vesicle fusion or monolayer transfer method, and (B) surface tethered vesicles. Planar membranes can be prepared by the surface grafting method, vesicle fusion, and monolayer trans-

fer methods.

wise synthesis of the polymer with a high molecular weight and a low polydispersity index. ${ }^{[35]}$ The 'grafting-from' approach is an efficient way to create 'hydrophilichydrophobic-hydrophilic' structures, which resemble biological membranes on solid supports. However, the dense packing of the polymer chains reduces the fluidity of the resulting membrane, and this generates steric constraints for protein incorporation.

A straightforward way to create planar membranes on surfaces is the process of vesicle fusion. For example, well-defined planar polymeric membranes were obtained using electrostatic interactions between positively charged poly(2,2-dimethylaminoethyl methacrylate)-block-poly(n-butyl methacrylate)-block-poly(2,2-dimethylaminoethyl methacrylate) (PDMAEMA$b$-PBMA- $b$-PDMAEMA) vesicles and negatively charged surfaces. ${ }^{[32 a]}$ The strength of the vesicle-substrate interaction and the nature of the membranes are modulated by the surface charge density. However, the cationic character of the polymer represents a limitation for technological applications that require functional insertion of biologically relevant species, such as peptides or proteins. The vesicle fusion approach can also be used for covalent immobilization of polymer membranes on solid supports, and the resulting membranes possess enhanced mechanical and chemical stability. We have reported the spreading of sulfur-functionalized poly(butadiene)-block-poly(ethylene oxide) (PB- $b$-PEO-LA) vesicles on gold substrate, ${ }^{[32 c]}$ but good control of packing density and surface coverage is not always feasible.

In order to control the ordering and packing of molecules, and thereby the thickness and architecture of the polymer layers, the most suitable preparation techniques are the Langmuir-Blodgett (LB) deposition ${ }^{[36]}$ and the Langmuir- 
Schaefer (LS) deposition. [37] Recently, we assembled - for the first time - two polymer layers to serve as solid-supported biomimetic membranes. ${ }^{[32 \mathrm{~b}]}$ A defect-free, homogeneous film of PB- $b$-PEO-LA was formed and immobilized on gold substrate by LB deposition, and this served as an anchoring layer on the solid support. A second layer of hydroxyl-functionalized poly(butadiene)-block-poly(ethylene oxide) (PB- $b$-PEO-OH) was then deposited by LS transfer; its attachment is mainly governed by hydrophobic interactions between the poly(butadiene) (PB) blocks. The assembled bilayers were uniform, fluid and defect-free, with a typically hydrophilic-hydrophobic-hydrophilic structure, and a thickness of $11 \mathrm{~nm}$.

Apart from planar solid-supported membranes, a tethering concept is used to immobilize vesicles on solid surfaces. Tethered vesicles represent ideal candidates for applications such as chemical/ biological reactions with precise control of the reaction environment, ${ }^{[38]}$ and encapsulation of active compounds in very small volumes for studies down to the single-molecule level.[39] The drawbacks of relatively high permeability and fragility of liposomes immobilized on solid substrates have been overcome by using stable polymeric vesicles. ${ }^{[40]}$

To support the tethering concept various interactions have been used, such as DNA hybridization, ${ }^{[41]}$ or biotin-streptavidin interaction. ${ }^{[38]}$ The modification of block copolymer membranes with specific recognition sites represents an elegant way for surface immobilization. Appropriately designed surface membranes, which contain molecules involved in biological recognition interactions, offer considerable potential for: i) fundamental investigations of this type of interaction, ii) as platforms to bind and sense the presence of specific molecules (proteins, enzymes, DNA), and iii) to create new materials where this type of interaction serves as a link between two different assemblies. For example, protein binding to metal-NTA functionalized block copolymer membranes on a solid support induces the formation of densely packed and ordered protein arrays that open a new route to induce $2 \mathrm{D}$ crystallization of proteins or to develop new types of biosensors. ${ }^{[42]}$ By designing two different polymersome-antibody conjugates using either anti-biotin IgG or trastuzumab, it was possible to specifically target biotinpatterned surfaces, which is an approach that appears promising for the development of new diagnostic materials. ${ }^{[15]}$

Surface-tethered polymer vesicles containing active compounds (e.g. enzymes, proteins, mimics) in their aqueous cavities allow the development of precise localized nanoreactors, which are essential for

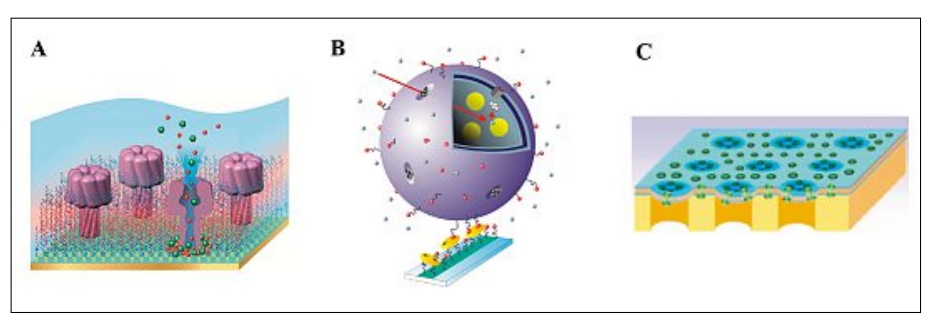

Fig. 4. Schemes of membrane constituted (A) solid-supported planar membranes, (B) surface tethered vesicles and (C) pore-solid-supported membranes.

medical applications, such as in implants or for patches. The first example of immobilized nanoreactors was introduced by an enzymatic reaction of acid phosphatase encapsulated in poly(2-methyl-2-oxazoline)block-poly(dimethylsiloxane)-blockpoly(2-methyl-2-oxazoline) vesicles that had been immobilized on a solid support via streptavidin-biotin interactions. OmpF inserted in the polymer membrane allowed the penetration of the substrate ELF97 to the inside of the nanoreactor and to support the acid phosphatase reaction in situ (Fig. 4B).[33] This system provided a first direct comparison between the activity of an encapsulated enzyme and that of the enzyme in free conditions.

Compared with solid-supported lipid membranes (with a hydrophilic/water layer of 1-3 nm), ${ }^{[43]}$ the thickness of polymer membranes ranges from 3 up to $40 \mathrm{~nm}$, depending on the hydrophilic and hydrophobic polymer blocks. These higher values of membrane thickness have the advantage of preventing strong interactions and/or frictional coupling between the solid substrate and incorporated proteins, which could result in partial loss of functionality or complete protein denaturation. ${ }^{[44]}$

By application of the Langmuir monolayer technique, it is possible to control the membrane film properties at the airwater interface, which is essential for studies of membrane protein insertion in a synthetic environment. For example, the miscibility (or phase separation) behavior of binary monolayers of PMOXAPDMS-PMOXA triblock copolymers and the antimicrobial peptide alamethicin, was investigated to find favorable conditions for insertion, and to obtain greater insight into the insertion process itself. ${ }^{[45]}$ Compared to lipid membranes, the larger polymer membrane possesses more flexibility and is able to adopt more conformations, and thus to support the hosting of a wider range of biomolecules in the membrane. Alternatively, mixed phase separated polymer-lipid thin films represent an interesting platform for more controlled and predictable location of membrane proteins. ${ }^{[46]}$ Interactions of poly(ethylene oxide)-block-poly(propylene oxide)block-poly(ethylene oxide) (PEO-b-PPO$b$-PEO) with various phospholipids ${ }^{[47]}$ and cell membrane have been studied. [48] Interestingly, the bacterial porin $\mathrm{OmpF}^{[46]}$ was found to be mainly present in the polymer-rich phase of the films based on mixtures of the PMOXA- $b$-PDMS- $b$-PMOXA triblock copolymer and 1,2-dipalmitoyl$s n$-glycero-3-phosphocholine.

Although the stronger interactions between polymer layers and substrates limit the mobility of polymers in solid-supported polymer bilayer membranes, the noncovalent interactions between two polymer layers allows a certain degree of membrane fluidity. This fluidity is essential for the insertion of peptides and membrane proteins, as we have established for insertion of the peptide, polymyxin B. However, polymyxin B did not form a transmembrane pore, but instead interacted with the polymer membrane in a detergent-like manner by creating transient defects and partly disassembling the membrane. ${ }^{[32 c]}$

Very recently, we reported the first functional incorporation of a water-soluble polypeptide, alpha-haemolysin in an artificial polymer tethered solid-supported bilayer membrane, TSSBM (Fig. 4A). ${ }^{[49]}$ The conductance variation upon channel protein insertion was modelled with Donnan potential, indicating that ions accumulated in the inner, hydrophobic part of the polymer membrane. Preliminary results indicated that the membrane protein $\mathrm{OmpF}$ was also functionally inserted, although in this case the presence of detergents was necessary. By substitution of one membrane protein by another, it will be possible to understand functional channel protein insertion, or to develop novel applications, such as biosensing, drug screening, or trace compound analysis.

Improvements of solid-supported polymer membrane systems can be achieved by forming pore-solid-supported membranes on porous pattern substrates. Such poresolid-supported membranes can probe the functionality of different transmembrane proteins (Fig. 4C), and are particularly attractive because they combine the mechanical stability of solid-supported membranes with the advantage of free-standing membranes. In addition, they offer unprecedented mechanical stability over periods of days with mesh sizes between $20 \mathrm{~nm}$ and several micrometers, in defined geometric patterns. A pore-solid-supported polymer membrane embedded with channel porin Aquaporin (AqpZ) has served to generate a highly permeable membrane that allows 
passive diffusion of small solutes such as ions, nutrients, or antibiotics. ${ }^{[50]}$ Moreover, high density reconstitution of functional AqpZ in a planar polymer membrane is achievable.[51]

\section{Conclusions}

Amphiphilic copolymers, which selfassemble into supramolecular structures, represent ideal candidates for forming $2 \mathrm{D}$ and $3 \mathrm{D}$ assemblies that can contain biomolecules or combinations thereof, because their chemistry allows the adjustment of properties such as stability, flexibility, and functionality. Up to now, most of the studies available on protein or peptide insertion into polymer membranes have been performed on vesicular systems, free-standing membranes, or monolayers at the air/water interface. Only recently, solid-supported polymer membranes have been introduced as templates for biomolecules to create 'active surfaces', which are particularly interesting for prospective technological applications, such as sensing, which may require the functional incorporation of proteins or peptides into the solid-supported polymer membranes.

Although there are many reported combinations of biomolecules with polymer membranes, there are still open questions to be solved because this is an emerging field. The reported systems are of interest from the fundamental point of view, and require further developments in terms of stability and functionality for potential applications.

Received: July 4, 2013

[1] a) S. M. Borisov, O. S. Wolfbeis, Chem. Rev. 2008, 108, 423; b) C. G. Palivan, O. FischerOnaca, M. Delcea, F. Itel, W. Meier, Chem. Soc. Rev. 2012, 41, 2800.

[2] X. Y. Zhang, P. Tanner, A. Graff, C. G. Palivan, W. Meier, J. Polym. Sci. Pol. Chem. 2012, 50, 2293.

[3] a) G. Battaglia, A. J. Ryan, J. Am. Chem. Soc 2005, 127, 8757; b) D. E. Discher, A. Eisenberg, Science 2002, 297, 967.

[4] M. Antonietti, S. Forster, Adv. Mater. 2003, 15, 1323.

[5] K. Kita-Tokarczyk, J. Grumelard, T. Haefele, W. Meier, Polymer 2005, 46, 3540.

[6] a) C. Schatz, S. Louguet, J. F. Le Meins, S. Lecommandoux, Angew. Chem. Int. Ed. 2009.
48, 2572; b) D. J. Adams, D. Atkins, A. I. Cooper, S. Furzeland, A. Trewin, I. Young, Biomacromolecules 2008, 9, 2997.

[7] T. B. Schuster, D. D. Ouboter, E. Bordignon, G. Jeschke, W. Meier, Soft Matter 2010, 6, 5596.

[8] E. G. Bellomo, M. D. Wyrsta, L. Pakstis, D. J. Pochan, T. J. Deming, Nat. Mater. 2004, 3, 244.

[9] a) E. Cabane, X. Y. Zhang, K. Langowska, C. G. Palivan, W. Meier, Biointerphases 2012, 7; b) X. K. Liu, M. Jiang, Angew. Chem. Int. Ed. 2006, $45,3846$.

[10] a) R. Stoenescu, W. Meier, Chem. Commun. 2002, 3016; b) Y. Matter, R. Enea, O. Casse, C. C. Lee, J. Baryza, W. Meier, Macromol. Chem. Phys. 2011, 212, 937.

[11] A. Blanazs, S. P. Armes, A. J. Ryan, Macromol. Rapid Comm. 2009, 30, 267.

[12] X. J. Li, I. V. Pivkin, H. J. Liang, G. E. Karniadakis, Macromolecules 2009, 42, 3195.

[13] V. A. Sethuraman, Y. H. Bae, J. Control Release 2007, 118, 216.

[14] P. Tanner, M. Ezhevskaya, R. Nehring, S. Van Doorslaer, W. Meier, C. Palivan, J. Phys. Chem. $B$ 2012, 116, 10113 .

[15] S. Egli, M. G. Nussbaumer, V. Balasubramanian, M. Chami, N. Bruns, C. Palivan, W. Meier, J. Am. Chem. Soc. 2011, 133, 4476.

[16] a) S. F. M. van Dongen, M. Nallani, S Schoffelen, J. J. L. M. Cornelissen, R. J. M. Nolte, J. C. M. van Hest, Macromol. Rapid Comm. 2008, 29, 321; b) D. A. Hammer, G. P. Robbins, J. B. Haun, J. J. Lin, W. Qi, L. A. Smith, P. P. Ghoroghchian, M. J. Therien, F. S. Bates, Faraday Discuss. 2008, 139, 129.

[17] a) P. Baumann, P. Tanner, O. Onaca, C. G. Palivan, Polymers 2011, 3, 173; b) D. E. Discher, F. Ahmed, Ann. Rev. Biomed. Eng. 2006, 8, 323.

[18] a) W. Meier, C. Nardin, M. Winterhalter, Angew. Chem. Int. Ed. 2000, 39, 4599; b) A. Ranquin, W. Versees, W. Meier, J. Steyaert, P. Van Gelder, Nano. Lett. 2005, 5, 2220.

[19] V. Malinova, S. Belegrinou, D. D. Ouboter, W. P. Meier, Adv. Polym. Sci. 2010, 224, 113.

[20] M. Kumar, M. Grzelakowski, J. Zilles, M. Clark, W. Meier, Proc. Natl. Acad. Sci. USA 2007, 104, 20719.

[21] A. Graff, C. Fraysse-Ailhas, C. G. Palivan, M. Grzelakowski, T. Friedrich, C. Vebert, G. Gescheidt, W. Meier, Macromol. Chem. Phys. 2010, 211, 229.

[22] a) C. Nardin, S. Thoeni, J. Widmer, M. Winterhalter, W. Meier, Chem. Commun. 2000, 1433; b) C. Nardin, J. Widmer, M. Winterhalter, W. Meier, Eur. Phys. J. E 2001, 4, 403.

[23] F. Axthelm, O. Casse, W. H. Koppenol, T. Nauser, W. Meier, C. G. Palivan, J. Phys. Chem. $B$ 2008, 112, 8211 .

[24] M. Nallani, S. Benito, O. Onaca, A. Graff, M. Lindemann, M. Winterhalter, W. Meier, U. Schwaneberg, J. Biotechnol. 2006, 123, 50.

[25] O. Onaca-Fischer, J. Liu, M. Inglin, C. G. Palivan, Curr. Pharm. Design 2012, 18, 2622.

[26] D. Dobrunz, A. C. Toma, P. Tanner, T. Pfohl, C. G. Palivan, Langmuir 2012, 28, 15889.

[27] V. Balasubramanian, O. Onaca, M. Ezhevskaya, S. Van Doorslaer, B. Sivasankaran, C. G. Palivan, Soft Matter 2011, 7, 5595.
[28] K. Langowska, C. G. Palivan, W. Meier, Chem. Commun. 2013, 49, 128.

[29] a) P. Tanner, O. Onaca, V. Balasubramanian, W. Meier, C. G. Palivan, Chem. Eur. J. 2011, 17, 4552; b) S. F. M. van Dongen, M. Nallani, J. J. L. M. Cornelissen, R. J. M. Nolte, J. C. M. van Hest, Abstr. Pap. Am. Chem. S 2009, 237.

[30] P. Tanner, V. Balasubramanian, C. G. Palivan, Nano. Lett. 2013, dx.doi.org/10.1021/ nl401215n.

[31] W. Senaratne, L. Andruzzi, C. K. Ober, Biomacromolecules 2005, 6, 2427.

[32] a) E. Rakhmatullina, W. Meier, Langmuir 2008, 24, 6254; b) S. Belegrinou, J. Dorn, M. Kreiter, K. Kita-Tokarczyk, E. K. Sinner, W. Meier, Soft Matter 2010, 6, 179; c) J. Dorn, S. Belegrinou, M. Kreiter, E. K. Sinner, W. Meier, Macromol. Biosci. 2011, 11, 514.

[33] a) M. Grzelakowski, O. Onaca, P. Rigler, M. Kumar, W. Meier, Small 2009, 5, 2545; b) T. Rosenkranz, A. Katranidis, D. Atta, I. Gregor, J. Enderlein, M. Grzelakowski, P. Rigler, W. Meier, J. Fitter, Chembiochem. 2009, 10, 702.

[34] T. Lehmann, J. Ruhe, Macromol. Symp. 1999, $142,1$.

[35] E. Rakhmatullina, T. Braun, T. Kaufmann, H. Spillmann, V. Malinova, W. Meier, Macromol. Chem. Phys. 2007, 208, 1283.

[36] K. B. Blodgett, J. Am. Chem. Soc. 1935, 57, 1007.

[37] I. Langmuir, V. J. Schaefer, J. Am. Chem. Soc. 1938, 60, 2803.

[38] E. Boukobza, A. Sonnenfeld, G. Haran, J. Phys. Chem. B 2001, 105, 12165.

[39] Y. H. M. Chan, S. G. Boxer, Curr. Opin. Chem. Biol. 2007, 11, 581.

[40] P. Vermette, L. Meagher, E. Gagnon, H. J. Griesser, C. J. Doillon, J. Control Release 2002, 80, 179.

[41] a) I. Pfeiffer, F. Hook, J. Am. Chem. Soc. 2004, 126, 10224; b) C. Yoshina-Ishii, G. P. Miller, M. L. Kraft, E. T. Kool, S. G. Boxer, J. Am. Chem. Soc. 2005, 127, 1356.

[42] R. Nehring, C. G. Palivan, O. Casse, P. Tanner, J. Tuxen, W. Meier, Langmuir 2009, 25, 1122.

[43] M. Tanaka, E. Sackmann, Nature 2005, 437, 656.

[44] E. K. Sinner, W. Knoll, Curr. Opin. Chem. Biol. 2001, 5, 705 .

[45] T. Haefele, K. Kita-Tokarczyk, W. Meier, Langmuir 2006, 22, 1164.

[46] J. Thoma, S. Belegrinou, P. Rossbach, M. Grzelakowski, K. Kita-Tokarczyk, W. Meier, Chem. Commun. 2012, 48, 8811.

[47] a) S. L. Frey, D. S. Zhang, M. A. Carignano, I Szleifer, K. Y. C. Lee, J. Chem. Phys. 2007, 127; b) E. Amado, J. Kressler, Curr. Opin. Colloid In. 2011, 16, 491.

[48] E. Amado, A. Blume, J. Kressler, React. Funct. Polym. 2009, 69, 450.

[49] X. Zhang, W. Fu, C. G. Palivan, W. Meier, Sci. Rep. UK 2013, 3, 2196.

[50] H. L. Wang, T. S. Chung, Y. W. Tong, K. Jeyaseelan, A. Armugam, Z. C. Chen, M. H. Hong, W. Meier, Small 2012, 8, 1969.

[51] M. Kumar, J. E. O. Habel, Y. X. Shen, W. P. Meier, T. Walz, J. Am. Chem. Soc. 2012, 134, 18631. 